\title{
A interface entre o PET-Saúde/ Interprofissionalidade e a Política Nacional de Educação Permanente em Saúde
}

\author{
The interface between Education through Work for Health Program/ \\ Interprofessionality and the National Policy of Permanent Education \\ in Health
}

Rodrigo Guimarães dos Santos Almeida', Elen Ferraz Teston'1, Arthur de Almeida Medeiros $\mathbf{1}$

DOI: $10.1590 / 0103-110420195108$

RESUMO Este ensaio teve como objetivo refletir sobre as contribuições da edição mais recente do Programa de Educação pelo Trabalho para a Saúde - PET-Saúde/Interprofissionalidade para a implementação da Política Nacional de Educação Permanente em Saúde (PNEPS) no âmbito do Sistema Único de Saúde. Nesse contexto, resgata-se a contemporaneidade da formação em saúde, a Educação Interprofissional e o processo formativo, e a execução do PET-Saúde/Interprofissionalidade como potencializador da prática colaborativa na atenção primária. Ressalta-se que, diante dos inúmeros desafios vivenciados pelos serviços de saúde para responder às demandas populacionais variadas e influenciadas pela transição demográfica e epidemiológica, entende-se como necessário ensaiar reflexões sobre a interprofissionalidade e suas contribuições para a implementação da PNEPS. Acredita-se que este material possa subsidiar a discussão e atuação profissional na elaboração e realização de intervenções interprofissionais apropriadas para as diferentes demandas em saúde.

PALAVRAS-CHAVE Serviços de integração docente-assistencial. Recursos humanos. Práticas interdisciplinares.

ABSTRACT This essay aims to reflect on the contributions of the most recent edition of the Education through Work for Health Program - PET-Health/Interprofessionality for the implementation of the National Policy of Permanent Education in Health (PNEPS) within the scope of the Unified Health System (SUS). In this context, the contemporaneity of health education, the Interprofessional Education and the formative process, and the implementation of PET-Health/Interprofessionality as a potentiator for the collaborative practice in primary care are rescued. It should be stressed that, given the innumerable challenges experienced by the health services to respond to the demands of a varied population and influenced by the demographic and epidemiological transition, we believe it is necessary to rehearse reflections on interprofessionality and its contributions to the strengthening of the PNEPS. It is believed that this material can subsidize the discussion and professional

1 Universidade Federal de Mato Grosso do Sul (UFMS) - Campo Grande (MS), Brasil.

rgclaretiano@gmail.com performance in the elaboration and accomplishment of interprofessional interventions that are appropriate for the different health demands.

KEYWORDS Teaching care integration services. Human resources. Interdisciplinary practices. 


\section{Introdução}

O contexto social contemporâneo, que se encontra marcado pelas intensas transformações de natureza política, científica, cultural, tecnológica e econômica, com impactos importantes na formação acadêmica, tem exigido das universidades e dos docentes a adoção de estratégias diferenciadas para o ensino de qualidade e que responda às necessidades não só do mercado de trabalho, mas também da sociedade.

As mudanças no perfil de morbimortalidade populacional têm evidenciado amplos desafios ao Sistema Único de Saúde (SUS), em especial no modo de cuidado centrado no paciente. Nesse contexto, emerge a necessidade do fortalecimento dos princípios básicos do SUS que oportunizam o cuidado integral e contínuo'.

Cabe destacar que os serviços de atenção à saúde, nos diferentes pontos da Rede de Atenção à Saúde (RAS), devem organizar a assistência em resposta às necessidades de saúde da população, que no cenário atual são representadas pela tripla carga de doenças: infecciosas e parasitárias, causas externas e condições crônicas, o que reitera os desafios vivenciados pelo setor. Atinente a isso, o descompasso entre a necessidade e a oferta das ações de promoção da saúde, prevenção de doenças, tratamento e reabilitação determinam as dificuldades atuais do SUS ${ }^{\mathbf{1} 2}$. Diante disso, todos os atores envolvidos no processo de formação devem estar abertos e aptos a compreender a necessidade da (re)construção diária de sua prática.

O ser/estar/tornar-se professor não se restringe unicamente ao ato de ensinar, é um processo construído no dia a dia da vivência da docência, que vai além do espaço físico da sala de aula, e que exige a mobilização e sistematização de inúmeros saberes. Ser docente universitário, principalmente na área da saúde, é uma nova experiência para todo profissional, visto que, conforme afirmam Pimenta e Anastasiou ${ }^{3(174)}$,

a maioria [das pessoas] que atua na docência universitária tornou-se professor da noite para o dia: dormiram profissionais e pesquisadores de diferentes áreas e acordaram professores.

$\mathrm{Na}$ área da saúde, a formação/preparação para o ensino se restringe, na maioria das vezes, às atividades desenvolvidas em disciplinas de didática, ou outras correlatas, cursadas na pós-graduação. Dessa maneira, a prática educativa de tais docentes baseia-se, principalmente, nas vivências experimentadas em sua caminhada de formação, as quais, muitas vezes, não são adequadas à realidade atual, e que serão, consequentemente, ofertadas aos estudantes e reproduzidas em suas vidas profissionais.

As Diretrizes Curriculares Nacionais (DCN) para os cursos da área da saúde definem como perfil do egresso: o profissional generalista, humanista, crítico, reflexivo, pró-ativo e criativo. Elas ainda elucidam que o processo de formação seja articulado, pautado e direcionado ao SUS e que garanta a atenção das reais necessidades de saúde da população ${ }^{4}$. Concernente a isso, torna-se imprescindível para a formação o reconhecimento das especificidades do território de atuação: o desenvolvimento de habilidades para o trabalho interprofissional, bem como o engajamento das diferentes profissões na discussão do cotidiano de trabalho.

Diante do apresentado, faz-se necessário romper com o modelo de formação tradicional, centrado em conteúdos e na pedagogia da transmissão, substituindo-o por metodologias de ensino-aprendizagem que oportunizem ao estudante ser o protagonista de sua formação. Essas novas metodologias devem ainda considerar a ampliação dos cenários de práticas pedagógicas para além do espaço de sala de aula, fortalecendo a integração entre o ensino, o serviço e a comunidade. Cabe salientar que o mundo do trabalho, no qual a vida acontece, deve ser encarado como espaço vivo de formação, em que conhecimentos, habilidades e atitudes são ensinados e vivenciados/aprendidos em ato, na vida cotidiana.

Considerando, portanto, as dificuldades do contexto social contemporâneo e as demandas 
emergentes do SUS, questiona-se: como formar profissionais comprometidos a gerir os problemas vivenciados na prática diária do trabalho e engajados com as especificidades/necessidades do serviço?

Posto isso, este ensaio tem como objetivo refletir sobre as contribuições da edição mais recente do Programa de Educação pelo Trabalho para a Saúde - PET-Saúde/ Interprofissionalidade para a implementação da Política Nacional de Educação Permanente em Saúde (PNEPS) no âmbito do SUS.

\section{Educação Interprofissional e o pro- cesso formativo}

Para pensar e refletir sobre o trabalho em saúde, é imprescindível discutir sobre a formação, com o objetivo de compreender os pontos de confluência e divergência dessas duas realidades, identificando as necessidades de (re) construção do processo formativo com foco nas reais demandas de saúde da população.

O modelo de formação hegemônico - hospitalocêntrico e fragmentado - apresenta uma valorização excessiva de competências técnicas específicas, o que contribui para a formação de profissionais com importantes limitações na capacidade de análise de contexto e de trabalhar colaborativamente em equipe $\mathrm{e}^{5,6}$.

A realidade de saúde da população, em que se verifica a complexidade do processo de cuidar, demonstra a necessidade do efetivo trabalho em equipe com práticas colaborativas e ações integradas centradas no usuário, na família e na comunidade. Nessa perspectiva, a Educação Interprofissional (EIP) mostra-se como uma importante ferramenta para a transformação do processo formativo, que visa à melhoria da colaboração e da qualidade da atenção à saúde. A EIP pode ser definida como um modelo de formação - estabelecido com ações articuladas entre dois ou mais cursos da saúde - que estimula o processo de aprendizagem compartilhado e interativo, em que se aprende sobre os outros, com os outros e entre si, necessária para o desenvolvimento de habilidades indispensáveis para a prática colaborativa $\mathbf{7}, \mathbf{8}$.

A EIP entrou em destaque no cenário nacional e internacional, em especial nos últimos anos, diante da urgente necessidade de (re) organização dos serviços de saúde perante as demandas existentes e do impacto positivo sobre a saúde da população, na qual observa-se um cuidado mais efetivo, integral, resolutivo, reduzindo os custos e os erros, e ampliando a segurança do paciente. A experiência da prática colaborativa entre os profissionais da saúde oportuniza a construção de um espaço rico para o desenvolvimento de atitudes, habilidades e conhecimentos indispensáveis para o trabalho interprofissional efetivo ${ }^{8}$.

No contexto da formação, a implementação da EIP ainda passa por diversos desafios e limitações; um deles está centrado na figura do docente, que necessita de qualificação relevante. Essa qualificação pode ser entendida como um conjunto de dimensões compostas por experiências prévias, intencionalidade para o trabalho em grupo colaborativo, flexibilidade e criatividade para vivenciar as situações de maneira compartilhada com os estudantes, e comprometimento ${ }^{8}$. Assim, é de grande importância uma reestruturação docente, ancorada na perspectiva da EIP, em que se ensina e se reflete sobre as experiências, construindo saberes, estratégias e projetos coletivos ${ }^{9}$.

Outro fator limitante é o suporte institucional, que ainda não valoriza a prática interprofissional e desconhece seus benefícios para integralidade do cuidado 9 . Para a subversão dessa lógica, é importante o fortalecimento da interação entre ensino, serviço e comunidade, extinguindo a cultura do ensino fragmentado e do trabalho individualizado.

Nesse contexto, torna-se premente o trabalho colaborativo entre os diferentes profissionais de saúde que atuam em prol da assistência resolutiva e de qualidade. Para tanto, são essenciais a prática reflexiva e o planejamento de ações a partir das lacunas identificadas no dia a dia do trabalho, por meio da utilização de uma 
abordagem coerente e integrada com os múltiplos fatores que influenciam o processo saúde-doença.

A prática colaborativa pode ser definida como uma cooperação integradora de diferentes profissionais de saúde, que combinam competências e habilidades em benefício do indivíduo - foco da atenção, possibilitando o melhor uso dos recursos disponíveis e o desenvolvimento de ações de cuidado mais criativas e efetivas ${ }^{10}$. Seus benefícios são inúmeros e confluem para o seu objetivo principal, qual seja: maior resolubilidade das práticas tendo em vista a melhoria dos resultados de saúde. Entretanto, a integração deficiente entre a tríade ensino, serviço e comunidade, mesmo com o apoio de políticas de nível macro, ainda se constitui uma barreira para sua implementação" ${ }^{11}$.

As experiências que avaliam a implementação da EIP, e, consequentemente, da prática colaborativa, apontam que a maioria das vivências são de curta duração (entre um e cinco dias) e que as limitações metodológicas são pouco exploradas. Tais estudos se centram na avaliação dos impactos da EIP apenas em curto prazo; e o dimensionamento das mudanças produzidas nas práticas profissionais ainda necessita de maior aprofundamento. Além disso, nas avaliações, embora os estudantes apresentem percepções positivas sobre a EIP, trabalhos que verifiquem a modificação de comportamento na prática profissional, mudança organizacional e melhoria da assistência ainda são pouco explorados ${ }^{8}$.

\section{A PNEPS como estratégia indutora para a prática colaborativa}

Como estratégia para transformar e qualificar a atenção à saúde, os processos formativos e as práticas de educação na saúde, por meio do incentivo à organização das ações e dos serviços em uma perspectiva intersetorial, pode-se destacar a PNEPS, que reafirma o compromisso do Estado brasileiro com o SUS, na garantia dos princípios de universalidade, equidade e integralidade com efetiva participação popular12-14.

A PNEPS apoia uma prática político-pedagógica voltada para ações de promoção, proteção e recuperação da saúde, por meio do diálogo entre a diversidade de conhecimento. Essa prática valoriza os saberes populares, a ancestralidade e o incentivo à produção individual e coletiva, inseridos no sistema público de saúde ${ }^{12-14}$. Tem como pilares o diálogo, a amorosidade, a problematização, a construção compartilhada do conhecimento, a emancipação e o compromisso com a construção do projeto democrático e popular. Entre os eixos estruturantes para a sua operacionalização, destacam-se a participação, o controle social e a gestão participativa, a formação, a comunicação, a produção de conhecimento, o cuidado em saúde, a intersetorialidade e os diálogos multiculturais ${ }^{12-14}$. Tais preceitos implicam a aproximação dos profissionais de saúde entre si e com a comunidade

Nesse contexto, a Educação Permanente em Saúde (EPS) constitui a inter-relação entre serviço, docência e saúde, com vistas ao desenvolvimento profissional e à qualidade da assistência prestada. Tem como base os pressupostos da aprendizagem significativa e da problematização da realidade, que devem ser orientadores das estratégias de mudança das práticas cotidianas de saúde ${ }^{15}$, no entendimento de que o trabalhador traz consigo conhecimento e experiências prévias e que o trabalho é fonte de conhecimento e local de aprendizagem. Nesse sentido, encoraja o uso de metodologias diferenciadas que despertem a reflexão crítica sobre as práticas de cuidado, adequando-as às reais necessidades dos usuários e atendendo aos princípios e diretrizes do SUS ${ }^{16}$.

Esse processo de análise rotineira das situações vivenciadas, que objetiva transformar as lacunas identificadas no trabalho em aprendizagem, é capaz de estimular a autonomia dos sujeitos e aproximá-los da 
realidade, oportunizando um processo educativo permanente ${ }^{16,17}$.

A gestão dos problemas vivenciados na prática é favorecida pelas ações de educação permanente, uma vez que elas possibilitam a ampliação da capacidade de autoavaliação pelos profissionais com relação às lacunas existentes entre o cenário ideal e o vivenciado no contexto dos serviços de saúde². Além disso, essas ações propiciam o engajamento dos profissionais com a reflexão sobre a prática diária e na não 'alienação' diante do contexto de trabalho, repercutindo positivamente na assistência prestada, visto que contribuem para a criação de novas possibilidades em detrimento da reprodução acrítica da realidade.

Ancorados na PNEPS, o Programa Nacional de Reorientação da Formação de Profissionais de Saúde (Pró-Saúde) e o PET-Saúde, em suas várias edições, sempre assumiram como eixo principal a integração ensino-serviço-comunidade, por meio do fortalecimento da parceria entre as Instituições de Ensino Superior (IES) e os serviços de saúde, com vistas a induzir processos de reorientação pedagógica nos cursos de saúde.

O PET-Saúde, na sua atual edição, apresenta como foco a interprofissionalidade, a interdisciplinaridade e a intersetorialidade, com o intuito de estimular a EIP em todo o território nacional. Seu objetivo é promover mudanças curriculares nos cursos de graduação na área da saúde alinhadas às DCN, com fundamentos para a mudança lógica da produção do cuidado, proporcionando qualificação dos processos de integração ensino, serviço e comunidade, de modo a suscitar as práticas colaborativas em saúde ${ }^{\mathbf{1 8}}$.

A prática colaborativa na perspectiva da EIP - pensada sob a ótica da aprendizagem colaborativa, experiencial e reflexiva - como uma ferramenta pedagógica, pauta-se na interação, colaboração e participação ativa dos envolvidos ${ }^{19}$. Trata-se de um processo de reaculturação, com o intuito de que essas ações se tornem parte da comunidade do conhecimento. Sua eficácia está no desenvolvimento do senso de trabalho em equipe e do senso crítico, lógico e analítico; na melhoria dos relacionamentos interpessoais; e no aprimoramento da comunicação, das habilidades de compreensão e da capacidade de lidar com pontos de vista diferentes ${ }^{20}$.

\section{O PET-Saúde/ Interprofissionalidade como potencializador da prática colaborativa na Atenção Primária à Saúde}

O PET-Saúde/Interprofissionalidade atende ao chamado da Organização Mundial da Saúde (OMS) para implementar a EIP como ferramenta essencial para o desenvolvimento e fortalecimento dos recursos humanos, contribuindo para a redução de muitos desafios enfrentados pelos sistemas de saúde no mundo ${ }^{\mathbf{7} 21}$. Nesse sentido, o PET-Saúde constitui-se uma estratégia de indução de mudanças no processo de formação profissional, possibilitando a identificação de necessidades frequentes vivenciadas no serviço de saúde e a reflexão por parte dos profissionais, a fim de originar propostas de intervenção contínuas ${ }^{22}$.

Estudo realizado na Paraíba, a partir de relatos oriundos das experiências de EPS desenvolvidas pelo grupo PET-Saúde, apontou que, embora os profissionais anseiem por melhoras na assistência, é nítida a dificuldade de discutir o processo de trabalho e de executar mudanças na prática. Entretanto, a adesão ao programa tem funcionado como potencializador para reflexões e envolvimento dos atores no processo de mudança ${ }^{23}$.

Vislumbra-se, a partir da proposta de implantação do PET-Saúde, a construção de estratégias de educação permanente como integrante do processo contínuo de trabalho, que pode ser favorecido por meio de parcerias com diferentes dispositivos de saúde da rede, como, por exemplo, os Núcleos Ampliados de Saúde da Família e Atenção Básica (Nasf-AB), a fim de fortalecer e favorecer a operacionalização das mudanças. 
Ele também visa reafirmar os pactos de universalidade, descentralização, integralidade e participação popular garantidos pelo SUS. Do ponto de vista histórico, sua execução vem reconfirmar, como política internacional, o compromisso firmado pelo Brasil para a Atenção Primária à Saúde (APS) na Declaração de Alma-Ata, que agora completa 40 anos.

Cabe destacar que a Declaração de AlmaAta representou um marco de cooperação entre os países, abarcando, inclusive, questões que ultrapassam o âmbito das condições de saúde. Ela buscou diminuir e amenizar as diferenças no desenvolvimento econômico e social entre os povos, sobretudo os mais necessitados, compreendendo que a promoção e a proteção da saúde são essenciais para o contínuo desenvolvimento e melhoria da qualidade de vida dos homens, o que, consequentemente, contribui para a paz mundial24.

A Declaração de Alma-Ata aponta a necessidade de compreensão da saúde como um direito humano e sugere, para a melhoria das condições de saúde, o apoio às instituições de serviços de saúde locais, centradas nas necessidades de saúde da população e da comunidade e fundamentadas na perspectiva interdisciplinar e na participação social. Ela apresenta como ações mínimas necessárias para o desenvolvimento da APS: educação em saúde voltada para a prevenção e proteção, distribuição de alimentos e nutrição apropriada, tratamento da água e saneamento, saúde materno-infantil, planejamento familiar, imunização, prevenção e controle de doenças endêmicas, tratamento de doenças e lesões e o fornecimento de medicamentos essenciais ${ }^{25}$.

$\mathrm{O}$ acordo firmado em Alma-Ata para o ano 2000 está longe de ser alcançado, tanto no mundo quanto no Brasil. No âmbito da saúde, é clara a necessidade de melhorar as condições de acessibilidade, de garantir os princípios de universalidade, integralidade e equidade e de diminuir as inúmeras iniquidades econômicas e sociais que influenciam diretamente as condições de saúde. Nesse sentido, os compromissos estabelecidos há 40 anos não se tornaram obsoletos; ao contrário, mostram-se atuais e cogentes, tendo sido reafirmados na Declaração de Astana, em $2018^{26}$.

O trabalho colaborativo é uma importante estratégia para ampliar a resolutividade na APS, principalmente tendo em vista os desafios vivenciados diariamente pelas equipes $\mathrm{da}$ Estratégia Saúde da Família para o cuidado em saúde. Sob esse aspecto, a criação do Nasf-AB configura-se como uma importante referência de apoio à reflexão da interprofissionalidade, cujo processo de trabalho se fundamenta na metodologia do apoio matricial, demandando a interação intra e interequipes e agregando, portanto, novos arranjos organizacionais ${ }^{27}$.

Destarte, visualiza-se o PET-Saúde/ Interprofissionalidade como recurso de estímulo à adoção de estratégias educacionais alicerçadas na EIP e na EPS e de promoção das práticas colaborativas, tendo como horizonte a consolidação da saúde como direito universal.

\section{Considerações finais}

Acredita-se que a implementação de políticas de nível macro sustentadas na integração ensino-serviço-comunidade tem potencial para fortalecer a EIP e implementação da PNEPS, ao estimular e conduzir processos de transformação das práticas de saúde em atendimento às reais necessidades da população usuária.

Nessa compreensão, espera-se que o PETSaúde/Interprofissionalidade, no âmbito do ensino, seja capaz de promover mudanças, tanto na forma de ensinar quanto na de aprender. Também se almeja que as IES possam compreender os novos rumos da educação na saúde e proporcionar apoio à comunidade acadêmica, seja na formação e aprimoramento dos docentes para EIP e suas bases metodológicas e conceituais, seja na criação de espaços de reflexão e trabalho coletivo, nos quais os discentes possam desenvolver competências para o trabalho em equipe interprofissional.

Para os serviços e para a comunidade, anseia-se que a atual edição do programa 
estimule o desenvolvimento de estratégias de EPS, especialmente quanto à prática colaborativa, objetivando qualificar a força de trabalho em saúde para a assistência centrada na pessoa e sua família, inseridos no seu ambiente, com todas as suas singularidades e encarados sob a perspectiva interdisciplinar, segundo a qual os saberes profissionais se complementam na busca pela integralidade do cuidado. Por consequência, almeja-se garantir práticas mais seguras e de maior qualidade, redução de erros profissionais e dos custos para o sistema de saúde, contribuindo, assim, para um SUS que realmente atenda às necessidades de seus utentes.

Como legado, a expectativa é que o PETSaúde/Interprofissionalidade possa tornar a prática colaborativa uma experiência que perpassa as interfaces entre a educação na saúde, o processo de trabalho e a prática profissional, não se tornando apenas uma vivência pontual, uma utopia, mas se concretizando e se perpetuando na busca constante pela qualificação do cuidado em saúde.

\section{Colaboradores}

Almeida RGS (0000-0002-4984-3928)* contribuiu para a concepção, o planejamento, a análise e a interpretação dos dados; revisão crítica do conteúdo; e aprovação da versão final do manuscrito. Teston EF (0000-0001-68350574)*, Medeiros AA (0000-0002-2192-8823)*. Contribuíram para a análise, interpretação dos dados; revisão crítica do conteúdo; e aprovação da versão final do manuscrito.

\section{Referências}

1. Mendes EV. O cuidado das condições crônicas na Atenção Primária à Saúde: o imperativo da consolidação da Estratégia da Saúde da Família. Brasília, DF: Organização Pan-Americana de saúde; 2012.

2. Massuda A, Hone T, Leles FAG, et al. Brazilian health system at crossroads: progress, crisis and resilience. BMG Glob Health [internet]. 2018 [acesso em 2018 out 20]; 3(4):e000829. Disponível em: https://www. ncbi.nlm.nih.gov/pmc/articles/PMC6035510/.

3. Pimenta SG, Anastasiou LGC. Docência no Ensino Superior. São Paulo: Cortez; 2002.

4. Brasil. Ministério da Saúde. Resolução CNS/MS n ${ }^{0}$ 569, de 8 de dezembro de 2017. Aprova o Parecer
Técnico $n^{\circ} 300 / 2017$ que apresenta princípios gerais a serem incorporados nas Diretrizes Curriculares Nacionais de todos os cursos de graduação da área da saúde, como elementos norteadores para o desenvolvimento dos currículos e das atividades didático-pedagógicas, e que deverão compor o perfil dos egressos desses cursos. Diário Oficial União. Brasília, DF, 26 Fev 2018.

5. Brasil. Ministério da Saúde. Relatório final da Oficina de alinhamento conceitual sobre Educação e Trabalho Interprofissional em Saúde. Brasília, DF: Ministério da Saúde; 2017.

6. Barr H, Low H. Introdução à Educação Interprofissioanl. Londres: CAIPE; 2013.
*Orcid (Open Researcher and Contributor ID). 
7. World Health Organization. Framework for Action on Interprofessional Education \& Collaborative Practice. Genebra, Suíça: WHO; 2010.

8. Reeves S. Why we need interprofessional education to improve the delivery of safe and effective care. Interface (Botucatu) [internet]. 2016 [acesso em 2018 out 27]; 20(56):185-197. Disponível em: http://dx.doi. org/10.1590/1807-57622014.0092.

9. Batista NA, Batista SHSS. Interprofessional education in the teaching of the health professions: shaping practices and knowledge networks. Interface (Botucatu) [internet]. 2016 [acesso em 2018 out 27]; 20(56):202-204. Disponível em: http://dx.doi. org/10.1590/1807-57622015.0388.

10. Supper I, Catala O, Lustman M, et al. Interprofessional collaboration in primary health care: a review of facilitators and barriers perceived by involved actors. J Public Health [internet]. 2015 [acesso em 2018 out 22]; 37(4):716-727. Disponível em: https://academic. oup.com/jpubhealth/article/37/4/716/2362834.

11. Silva JAM, Peduzzi M, Orchard C, et al. Educação interprofissional e prática colaborativa na Atenção Primária à Saúde. Rev. Esc. Enferm. USP [internet]. 2015 [acesso em 2018 out 23]; 49(spe2):1624. Disponível em: http://dx.doi.org/10.1590/ S0080-623420150000800003.

12. Brasil. Ministério da Saúde. Portaria nº 198, de 13 de fevereiro de 2004. Institui a Política Nacional de Educação Permanente em Saúde como estratégia do Sistema Único de Saúde para a formação e o desenvolvimento de trabalhadores para o setor e dá outras providências. Diário Oficial União. Brasília, DF, 16 Fev 2004.

13. Brasil. Ministério da Saúde. Portaria no 2761, de 19 de novembro de 2013. Institui a Política Nacional de Educação Permanente em Saúde como estratégia do Sistema Único de Saúde. Diário Oficial União. Brasília, DF, 19 Nov 2013.

14. Brasil. Ministério da Saúde. Portaria n ${ }^{\circ} 11$, de 17 de janeiro de 2017. Estabelece o Plano Operativo para implementação da Política Nacional de Educação Popular em Saúde no âmbito do Sistema Único de Saúde (PNEPS-SUS). Diário Oficial União. Brasília, DF, 17 Jan 2017.

15. Pessôa LR, Ferla AA, Andrade J, et al. A educação permanente e a cooperação internacional em saúde: um olhar sobre a experiência de fortalecimento da rede haitiana de vigilância, pesquisa e educação em saúde, no âmbito do projeto Tripartite Brasil-HaitiCuba. Saúde debate [internet]. 2013 [acesso em 2018 out 22]; 49:165-71. Disponível em: http://www.ensp. fiocruz.br/portal-ensp/informe/site/arquivos/anexo s/63802503ec0a8c6a0118ff263102e43216771f9f.PDF.

16. Brasil. Ministério da Saúde, Secretaria de Gestão do Trabalho e da Educação na Saúde, Departamento de Gestão da Educação em Saúde. Política Nacional de Educação Permanente em Saúde. Brasília, DF: Ministério da Saúde; 2009.

17. Melo MC, Queluci GC, Gouvea MV. Problematizing the multidisciplinary residency in oncology: a practical teaching protocol from the perspective of nurse residents. Rev Esc Enferm USP [internet]. 2014 [acesso em 2018 set 18]; 48(4):706-14. Disponível em: http://www.scielo.br/pdf/reeusp/v48n4/0080-6234reeusp-48-04-706.pdf.

18. Brasil. Ministério da Saúde. Edital no 10, 23 de julho 2018 seleção para o Programa de Educação pelo Trabalho para a Saúde PET-Saúde/ Interprofissionalidade - 2018/2019. Diário Oficial União. Brasília, DF, 24 Jul 2018.

19. Sargeant J. Theories to aid understanding and implementation of interprofessional education. J Contin Educ Health Prof. [internet]. 2009 [acesso em 2018 set 18]; 29(3):178-84. Disponível em: http://dx.doi. org/10.1002/chp.20033.

20. Johnson DW, Johnson RT, Smith KA. Cooperative learning returns to college: what evidence is there that it works? Change [internet]. 1998 [acesso em 2018 set 18]; 30(4):26-35. Disponível em: https://studentsuccess.louisiana.edu/sites/studentsuccess/files/ CooperativeLearning.pdf. 
21. Silva FAM, Cassiani SHB, Freire Filho JR. Interprofessional Health Education in the Region of the Americas. Rev. Latino-Am. Enfermagem [internet]. 2018 [acesso em 2018 set 20]; 26:e3013. Disponível em: http://dx.doi.org/10.1590/1518-8345.0000.3013.

22. França T, Magnago C, Santos MR, et al. PET-Saúde/ GraduaSUS: retrospectiva, diferenciais e panorama de distribuição dos projetos. Saúde debate [internet]. 2018 [acesso em 2018 dez 21]; 42(spe2):286-301. Disponível em: https://scielosp.org/article/sdeb/2018. v42nspe2/286-301/pt/.

23. Sampaio J, Santos KSD, Carvalho POF, et al. Pet-Saúde e Educação Permanente: dispositivos potentes na problematização do acolhimento na Saúde da Família. Rev. Bras. Ciênc. Saúde [internet]. 2014 [acesso em 2018 dez 21]; 18(spl1):49-54. Disponível em: http:// www.periodicos.ufpb.br/ojs/index.php/rbcs/article/ view/20941/11843.

24. Mendes IAC. Desenvolvimento e saúde: uma declaração de Alma-Ata e movimentos posteriores. Rev.
Latino-Am. Enfermagem [internet] 2004 [acesso em 2018 out 29]; 12(3):447-48. Disponível em: http:// dx.doi.org/10.1590/S0104-11692004000300001.

25. Declaração de Alma-Ata. Conferência Internacional sobre Cuidados Primários em Saúde; 6-12 Set. 1978; Alma-Ata (URSS): WHO; 1978.

26. Declaration of Astana. Global Conference on Primary Health Care, 25-26 Out. 2018; Astana (Kazakhstan): WHO; 2018.

27. Bispo Júnior JP, Moreira DC. Cuidado colaborativo entre os núcleos de apoio à saúde da família e as equipes apoiadas. Physis [internet] 2018 [acesso em 2018 out 29]; 28(3):e280310. Disponível em: http:// www.scielo.br/pdf/physis/v28n3/0103-7331-physis28-03-e280310.pdf.

Recebido em 26/12/2018 Aprovado em 07/05/2019

Conflito de interesses: inexistente

Suporte financeiro: não houve 